\title{
Quantification in cardiac MRI: advances in image acquisition and processing
}

\author{
Anil K. Attili • Andreas Schuster • Eike Nagel • \\ Johan H. C. Reiber • Rob J. van der Geest
}

Received: 10 November 2009/Accepted: 18 December 2009/Published online: 8 January 2010

(C) The Author(s) 2010. This article is published with open access at Springerlink.com

\begin{abstract}
Cardiac magnetic resonance (CMR) imaging enables accurate and reproducible quantification of measurements of global and regional ventricular function, blood flow, perfusion at rest and stress as well as myocardial injury. Recent advances in MR hardware and software have resulted in significant improvements in image quality and a reduction in imaging time. Methods for automated and robust assessment of the parameters of cardiac function, blood flow and morphology are being developed. This article reviews the recent advances in image acquisition and quantitative image analysis in CMR.
\end{abstract}

A. K. Attili

Department of Radiology and Cardiology, University of Kentucky, 800 Rose Street Room HX-315B,

Lexington, KY 40536, USA

A. Schuster - E. Nagel

King's College London BHF Centre of Excellence, Division of Imaging Sciences, NIHR Biomedical Research Centre at Guy's and St. Thomas' NHS Trust Foundation, The Rayne Institute, 4th Floor Lambeth Wing, St. Thomas' Hospital, London SE1 7EH, UK

J. H. C. Reiber · R. J. van der Geest $(\bowtie)$

Division of Image Processing, Department of Radiology, Leiden University Medical Center, Albinusdreef 2,

2333 ZA Leiden, The Netherlands

e-mail: R.J.van_der_Geest@lumc.nl
Keywords Cardiac MRI - Quantification

$\begin{array}{ll}\text { Abbreviations } \\ \text { 2D } & \text { Two-dimensional } \\ \text { 3D } & \text { Three-dimensional } \\ \text { CAD } & \text { Coronary artery disease } \\ \text { CMR } & \text { Cardiac magnetic resonance } \\ \text { CSPAMM } & \text { Complementary spatial modulation of } \\ & \text { magnetization } \\ \text { DENSE } & \text { Displacement encoding with stimulated } \\ & \text { echoes } \\ \text { DE-MR } & \text { Delayed-enhancement magnetic reso- } \\ & \text { nance } \\ \text { ED } & \text { End-diastolic } \\ \text { EF } & \text { Ejection fraction } \\ \text { EPI } & \text { Echo-planar imaging } \\ \text { ES } & \text { End-systolic } \\ \text { GRE } & \text { Gradient-echo } \\ \text { HCM } & \text { Hypertrophic cardiomyopathy } \\ \text { LV } & \text { Left ventricle } \\ \text { MBF } & \text { Myocardial blood flow } \\ \text { MR } & \text { Magnetic resonance } \\ \text { RV } & \text { Right ventricle } \\ \text { SENC } & \text { Strain-encoding } \\ \text { SENSE } & \text { Sensitivity encoding } \\ \text { SNR } & \text { Signal to noise ratio } \\ \text { SSFP } & \text { Steady-state free precession } \\ \text { SPAMM } & \text { Spatial modulation of magnetization } \\ \text { VEC } & \text { Velocity-encoded cine } \\ & \end{array}$




\section{Introduction}

The diagnosis and management of cardiac disease requires a precise assessment of the parameters of cardiac morphology and function. Cardiac magnetic resonance (CMR) imaging has shown to be a versatile non-invasive imaging modality providing accurate and reproducible assessment of global and ventricular regional function, blood flow, myocardial perfusion and myocardial scar. In addition to enhancing clinical decision making, the accuracy and reproducibility of the CMR quantitative measures of cardiac function and morphology allow research studies to be carried out with fewer subjects enhancing cost effectiveness. Significant recent advances have been made in the generation of new CMR acquisition protocols as well as MR hardware enabling more rapid image acquisition. Despite these advances, the quantitative analysis of the images often still relies on manual tracing of the contours in many images, a time-consuming process. Reliable automated or semi-automated image segmentation and analysis software allowing for reproducible and rapid quantification are under development. In this paper an overview is provided on some of the recent work that has been carried out on image acquisition, computerized quantitative image analysis methods and semi-automated contour detection software for CMR imaging. The emerging clinical applications of quantitative CMR parameters are highlighted.

\section{Assessment of global ventricular function}

The accurate and reproducible quantification of left and right ventricular volumes, function and mass is crucial for the determination of appropriate therapeutic procedures, monitoring disease progression/ response, timing of surgery and prognostic stratification in patients with cardiac disease. CMR is recognized as the reference standard for the assessment of left and right ventricular volume and mass; it has been shown to be accurate, highly reproducible and without geometric assumptions [1]. Measurements of global ventricular function are typically derived from a stack of multi-slice cine 2D steady state free precession (SSFP) images acquired during multiple periods of breath-holding. The increasing image acquisition speed in recent years has been associated with stronger magnetic field gradients. The advent of parallel imaging has made available additional speeding up of data acquisition by exploiting the characteristics of antenna arrays for signal reception. In parallel imaging only, a subset of data required to reconstruct the full image is encoded by the magnetic gradient action. The missing information is repopulated, based on differences in perception of the object signal by multiple receiver antennas placed around the object. Among all the different parallel imaging techniques presented to date, SENSE [2] and GRAPPA [3] have been the most successful and are now widely available on commercial MR systems. Central to parallel imaging is the availability of dedicated receiver array coils. The typical speed up factors achievable in cine cardiac imaging with the standard five element cardiac coil arrays range from two to four. Beyond this range SNR limitations increasingly affect the image quality. Several studies have shown that cine imaging with SENSE and other parallel imaging methods yields comparable measurements of left and right ventricular volume and function to conventional cine imaging [4].

In addition to using parallel imaging, a different approach to faster imaging of dynamic objects is based on the observations of the correlation of image information in space and time. In a cine image of the heart, large regions of the image such as the chest wall and liver are static or only moderately dynamic. In addition, individual neighboring time frames of the heart are very similar suggesting that considerable information redundancy is present in the data. Accordingly, an optimized acquisition scheme would need to update highly dynamic information at a high rate, whereas less dynamic or static information can be acquired at a much lower rate. Among such techniques k-t BLAST (Broad-use Linear Acquisition Speed-up Technique) and k-t SENSE (SENSitivity Encoding) are widely available and have received widespread attention [5].

Both methods are based on the observation that dynamic data sets exhibit considerable correlation in space and time. These so called under-sampling techniques take advantage of this correlation by only acquiring a subset of the data and recovering the missing data points in the subsequent reconstruction process. In k-t BLAST and k-t SENSE the image content is estimated based on the so-called training 
data that serves as guidance for image reconstruction of the missing information. The k-t BLAST and k-t SENSE techniques typically permit a five-fold to eight-fold acceleration in dynamic cine imaging of the heart. Increased scan efficiency with these reduced data acquisition methods may be used to facilitate single-breath-hold 3D cine imaging. Single breath-hold k-t BLAST accelerated time resolved 3D cine imaging has been shown to provide accurate assessment of left and right ventricular volume and mass when compared to the standard multi-slice, multiple breath-hold SSFP imaging [6, 7]. The potential benefits of 3D cine imaging include easier planning, more rapid data acquisition and reconstruction in arbitrary planes as well as avoidance of misregistration artifacts that can affect multi-slice $2 \mathrm{D}$ imaging with multiple breath-holds. 3D cine imaging using k-t BLAST has also been successfully applied to dobutamine stress MR [8].

More rapid techniques continue to be developed. A higher level of acceleration without SNR limitations is possible by the wider availability of state of the art 32-element coil arrays with even more channels available in the near future [9]. In addition, newer intravascular MRI contrast agents [10] and higher field MR [11] combined with faster image acquisition techniques and new coil developments may help to increase image contrast, reduce motion artifacts and further decrease image acquisition times.

\section{Quantitative image analysis for assessment of global function}

Assessment of global function requires the measurement of the chamber volumes at different points in the cardiac cycle. Single or bi-plane long-axis views may be used in cases with little time available for image acquisition and image review. In normal volunteers and selected patients, good agreement has been observed with results derived from multislice short-axis acquisitions [12]. The advantage of using a multi-slice short-axis acquisition is that the LV wall is imaged almost perpendicularly, resulting in minimal partial volume averaging artifacts, and in addition, the measurements are independent of geometrical assumptions. However, with the currently employed slice thickness values of $5-10 \mathrm{~mm}$, a limitation is the relatively poor depiction of the most basal section. The significant myocardial motion in the through-plane direction is another factor contributing to the complexity of the analysis at the most basal section. For normal ventricles, the basal through-plane motion exceeds the typical section thickness [13]. For global RV function quantification, it has been shown that the use of axial slices has advantages over the short-axis orientation [14]. Quantification of volumetric dimensions requires segmentation of the acquired MR images. The segmentation process involves the isolation of the blood pool of the ventricle in multiple images. For each phase in the cardiac cycle, the blood volume is derived from the segmentation by multiplying the cross-sectional area of the blood pool in each imaging section by the sum of the section thickness and interslice gap.

As manual image segmentation is a time-consuming and tedious process many studies have attempted to develop an automated or semi-automated image segmentation approach. Most of the successful contour detection methods are based on underlying geometrical or statistical models. The model is used to constrain the segmentation of the LV to realistic shapes, having the advantage that the segmentation process is less sensitive to image artifacts. Examples of model based segmentation methods are the methods based on Active Shape Models (ASM) [15] and Active Appearance Models (AAM) [16, 17]. Both 2and 3-dimensional models are currently being used. Since every image slice is usually acquired during another breath-hold, the actual image set does not really form a true $3 \mathrm{D}$ scene; therefore, application of a 3D model requires a pre-processing step in which the image slices are correctly aligned. A disadvantage of using a geometrical model is that in cases with ventricular pathology resulting in strange shapes, the segmentation may be erroneous at particular regions. AAM methods can also be used to model the geometric deformation of the myocardium over the cardiac cycle. Contour detection using the $2 \mathrm{D}+$ time AAM method is less sensitive to image artifacts in individual image frames, since the temporal smoothness of the contours is an intrinsic property of the algorithm [18].

Inspired by the significant improvement in image quality, more recent automated segmentation methods follow a more data-driven approach [19, 20]. The work by Codella et al. describes an elegant 
segmentation method that takes into account the partial volume effect that occurs with the typical slice thickness values used. In this method, pixels within the LV endocardial boundary partially contribute to the blood volume and partially to the myocardial mass, depending on the pixel intensity. As such, this approach accounts for small trabeculations and papillary muscles. For assessment of the LV mass the epicardial contours also need to be defined. Automated detection of the epicardial boundaries is more complex due to the varying image intensities of the surrounding structures (e.g., lungs, diaphragm, RV blood, epicardial fat). In general, model-based segmentation methods prove to be more successful for this.

\section{Evaluation of regional ventricular function}

Myocardial strain imaging using tagged cine MRI involves the assessment of local tissue deformation as an indicator of regional myocardial contractile function. MR tagging is performed with cine MR imaging by applying a special radio-frequency pre-pulse immediately following detection of the $\mathrm{R}$ wave on the ECG tracing [21]. The pre-pulse is oriented perpendicular to the imaging plane and induces a local saturation that is depicted on the images as a dark line superimposed on the myocardial tissue. The multiple lines produced by successive applications of the pre-pulse are observed as parallel stripes or as a grid on the cine MR images of the cardiac cycle. MR tagging is conventionally performed by using segmented k-space spoiled gradient echo pulse sequences typically with a spatial modulation of the magnetization (SPAMM) technique [22]. Short-axis and long-axis two-dimensional tagged images allow the tracking of the myocardial strain in three dimensions. MR tags usually fade in early diastole (approximately 400$500 \mathrm{~ms}$ ) because of $\mathrm{T} 1$ relaxation. A technique that improves tag persistence and can be used for tagging in both systole and diastole is complementary SPAMM (CSPAMM) [23]. With the CSPAMM method, two acquisitions are subtracted one from the other: one obtained with a SPAMM technique and a second obtained with a saturation pre-pulse that is inverted with respect to the SPAMM sequence. The primary drawback of the CSPAMM technique is that it doubles the acquisition time and thus the breath-holding time, which may result in the misregistration of the images to be subtracted. However, a reduction of imaging time with CSPAMM is possible by applying a segmented echo-planar imaging (EPI) sequence that allows the acquisition of systolic and diastolic grid-tags within a single breath-hold [24]. The EPI CSPAMM technique with a shortened acquisition time has been used for myocardial tagging under physical stress at high heart rates [25]. Spoiled gradient echo imaging has most commonly been used for signal generation; however, recent studies have demonstrated better tag contrast and persistence with the SSFP read-outs [26].

Recent developments in MR tagging include the development of inherent tissue tracking methods without tag detection and straightforward analysis such as harmonic phase imaging (HARP), displacement encoding with simulated echoes (DENSE) and strain-encoded (SENC) MR. HARP and DENSE techniques encode myocardial tissue displacement into the phase of the MRI signal and then compute myocardial strain from phase-reconstructed images [27]. Pixel-wise high spatial resolution and inherent tissue tracking are achieved.

Strain-encoded (SENC) imaging has recently been introduced as a new method for measuring myocardial strain [28]. In contrast to the SPAMM conventional tagging, SENC uses tag surfaces parallel to the image plane combined with out-ofplane phase-encoding gradients along the sliceselected direction. Radiofrequency (RF) pulses with ramped flip angles are applied to compensate for the tag fading caused by longitudinal relaxation and to maintain constant myocardial signal intensity throughout the cardiac cycle. By combining SENC magnitude images at two different tuning frequencies (low- and high-tuning), a SENC strain map is computed, which is then overlaid pixel-by-pixel on the anatomic image long-axis views that have to be generated to calculate circumferential strain, and short-axis views to measure the longitudinal strain. This technique produces images where the intensity depends on the degree of tissue deformation, measured by the strain (i.e., the change in length per unit length of tissue). Therefore, the resulting anatomical images of the scanner are encoded with the strain values of the deformations. No postprocessing of the resultant maps is needed when this method is used. SENC provides a higher temporal 
and spatial resolution of strain measurements throughout the whole cardiac cycle and has been shown to be a reliable tool for accurate and objective quantification of regional myocardial systolic and diastolic function when compared to conventional SPAMM tagging [29]. A dysfunctional region on SENC images corresponds with the region of hyperenhancement on delayed contrast-enhanced images in patients with a myocardial infarction (MI) [30]. The direct color-coded visualization of the myocardial strain rate with the SENC rate has been shown to be a useful adjunct for the dobutamine stress MR (DSMR); it provides incremental value for the detection of CAD compared to conventional wall motion readings on cine images [31]. A further modification of the SENC technique known as the fast-SENC pulse sequence, enables one to acquire strain-encoded images in a single heartbeat by combining localized SENC, spiral imaging, and interleaving of the individual components used for SENC imaging [32]. Real time imaging of regional myocardial function is made possible [33]. The SENC technique has been shown to be feasible for the detection of regional right ventricular strain due to its high image quality and spatial resolution [34, 35]. The use of conventional methods of MR tagging in imaging of the right ventricle is limited because of the thinness of the myocardium and the orientation of the myocardial strain in the right ventricle.

Regional ventricular function may also be assessed from standard cine MR sequences, based on the evaluation of the movement of the endocardial boundary, or the thickening of the myocardium during systole. Once endocardial and epicardial contours are available for the ED and ES phases, the centerline method can be employed to quantify regional function. Since this method is only capable of assessing the radial component of myocardial motion, it is less advanced than the previously described MR techniques. By analyzing the evolution of the segmental myocardial thickness or myocardial motion over the complete cardiac cycle, it can also be used to assess LV dys-synchrony for prediction of the response to cardiac resynchronization therapy [36, 37]. Figure 1 illustrates the results from temporal segmental wall motion analysis in a patient with and a patient without wall motion dyssynchrony.

\section{Evaluation of blood flow in the heart and great vessels}

Cardiac-gated GRE sequences with flow-encoding gradients are used to quantify velocity and blood flow in the heart and great vessels. These sequences are referred to as velocity encoded cine (VEC) MRI or phase-contrast MRI. 2D VEC MRI sequences are the most commonly used in clinical practice. They can be used to quantify cardiac output, pulmonary to systemic flow ratio, valvular regurgitation, and coronary flow reserve. In addition, the location and severity of flow obstruction can be observed. VEC MRI is useful for corroborating volumetrics obtained with cine imaging of the heart to assure that the data obtained are accurate.

Newer VEC MRI sequences allow resolution of velocity vectors in three directions, with spatial coverage of a 3D volume, temporally resolved throughout the cardiac cycle. Such sequences have been coined 7D flow encoding and have the advantage of providing complete spatial and temporal resolution of velocity with a higher signalto-noise ratio than 2D methods [38, 39]. Such acquisitions may be used for visualization of flow patterns in the heart and great arteries or for more accurate quantification of blood flow. Westenberg et al. demonstrated that such VEC MRI sequences can be used to obtain more accurate assessment of trans-valvular flow and used this technique in volunteers and patients with mitral and tricuspid valve regurgitation [40]. Quantification of transvalvular flow from these 3D acquisitions involves retrospective definition of the valve plane. After reconstruction of the through-plane component of flow through the valve orifice, trans-valvular flow can be assessed using standard techniques at each point in the cardiac cycle.

\section{Myocardial perfusion at rest and stress}

CMR perfusion imaging can quantify the absolute myocardial blood flow (MBF) in milliliters/minute/ gram. Some advantages of CMR perfusion imaging include its lack of ionizing radiation, the wide availability of CMR imaging systems, and a sufficiently high spatial resolution to allow analysis of transmural differences in myocardial blood flow. 

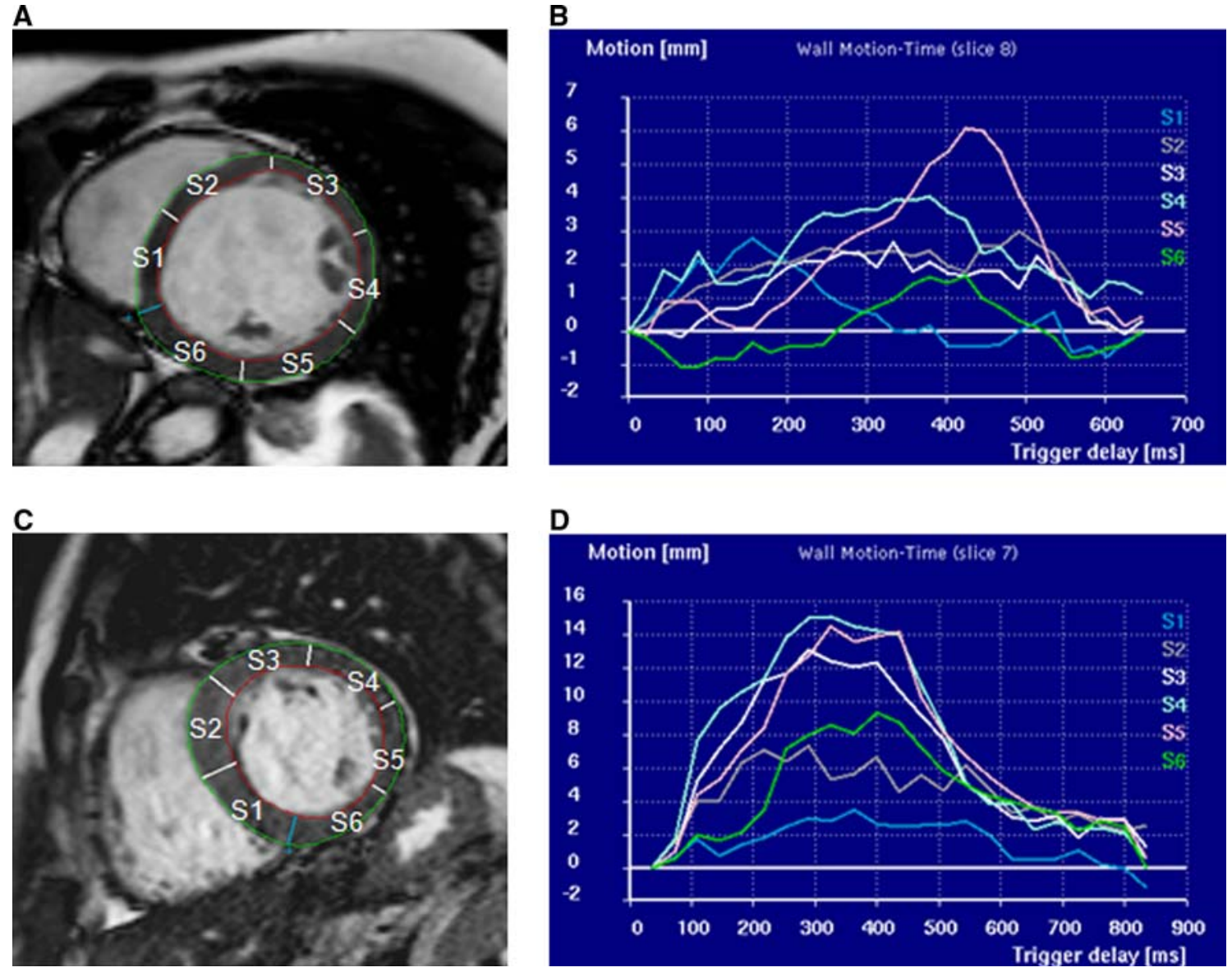

Fig. 1 a, b Patient with dyssynchronous myocardial wall motion as clearly revealed by the uncoordinated wall motion pattern derived from the endocardial contours detected in every

image frame. c, d Patient with normal synchronous wall motion as indicated by the normal temporal wall motion curve

Significant technical and clinical advances have occurred in the field of CMR perfusion imaging in recent years. The diagnostic accuracy of the technique has been validated against competing non invasive and invasive modalities in single center and increasingly in multicenter studies [41, 42]. In addition, increasing prognostic data on the value of stress perfusion MR has become available [43]. As a result, stress perfusion MRI has evolved from a promising research tool to an everyday clinical test. The ability to detect global reductions in perfusion reserve, to assess serial changes in flow with improved precision, and to examine subendocardial flow can provide important insights into our understanding of the pathophysiology of myocardial disease and aid in the evaluation of novel therapies.

\section{CMR perfusion imaging acquisition}

The use of T1-sensitive imaging sequences during the first pass bolus injection of a gadolinium-based contrast agent is the most robust and extensively studied CMR technique available for assessing myocardial perfusion. T1-weighting can be obtained by inversion recovery or saturation recovery, either nonslice selective or slice-selective (=notched pulse saturation). The main issue in the design of CMR perfusion sequences is the trade-off between spatial and temporal resolution. A sufficient temporal and spatial resolution, which is required to image several cardiac slices, every or at least every other heartbeat, and to achieve an in-plane resolution of $2-3 \mathrm{~mm}$ to separately visualize the endocardial and epicardial layers, can be obtained with a fast data readout by 
means of gradient echo (GRE), echo planar imaging (EPI) or SSFP techniques [44]. Although there is no standardized technical approach, the use of a balanced SSFP technique has been shown to provide higher peak enhancement and superior image quality when compared to other sequences, when using $0.05 \mathrm{mmol} / \mathrm{kg}$ body weight of Gd-BOPTA [45]. Typically, three to five short-axis slices of the heart are acquired over 5-10 s prior to the injection of the intravenous contrast bolus, and about $60 \mathrm{~s}$ after the injection of contrast. Imaging during pharmacological vasodilatation with adenosine or dipyridamole is routinely performed to improve differentiation of normal from stenotic perfusion beds. The regional image contrast enhancement should ideally be proportional to the contrast agent concentration. Such an approximate linear relationship between regional signal intensity and contrast agent concentration is only observed at lower contrast agent doses. Dosage regimes for gadolinium based extracellular contrast agents have varied between 0.025 and $0.15 \mathrm{mmol} / \mathrm{kg}$ in different studies. Investigators have also used a double bolus technique for first pass CMR perfusion rather than a single bolus for quantitative analysis of perfusion [46]. This is performed by giving a very low dose bolus initially to generate arterial input function, an indication of the tracer available for coronary perfusion, followed by a high dose bolus to maximize myocardial enhancement. The double bolus technique has been suggested as providing less distortion of the left ventricular cavity signal intensity, thereby allowing accurate depiction of the arterial input function. Quantitative myocardial perfusion using a double bolus first pass perfusion has been validated in animal models against microspheres, and has been shown to differentiate hyperemic blood flow in healthy human subjects.

\section{Qualitative and Semi-quantitative analysis}

In CAD diagnosis of a hemodynamically significant stenosis and assessment of functional significance of an anatomical lesion form an important part of clinical management. The simplest method for interpreting CMR perfusion studies is to view the study in cineloop format for regions of relative hypoperfusion. This is the most common method used in clinical practice and both single and multicenter studies have validated the diagnostic accuracy of the visual assessment of stress perfusion data against invasive angiography [41]. A number of semi-quantitative parameters can also be obtained from the time intensity curve obtained by plotting the signal intensity (SI) against time from the dynamic perfusion images. These parameters include the maximal signal intensity, contrast appearance time, time to maximal SI, area under the SI curve and the steepness of the SI curve's upslope determined by a linear fit to the initial part of the curve. Of these parameters the upslope of the SI curve has been most widely adopted for semiquantitative analysis and has been shown to improve diagnostic accuracy over visual analysis alone [47]. A recent meta-analysis showed an overall sensitivity of $91 \%$ and specificity of $81 \%$ for CMR perfusion imaging using qualitative and semi-quantitative analysis for the diagnosis of CAD, compared to quantitative coronary angiography [41].

\section{Image processing of perfusion imaging studies}

To derive quantitative indices related to the presence or absence of myocardial perfusion deficits, timeintensity curves must be evaluated for regions within the myocardium. Due to the significant patient motion over the acquisition period, time-intensity curves that are derived from a static region in the image are severely distorted (Fig. 2). Automated image co-registration techniques have been developed to correct for motion artifact [48-50]. Once the images are registered, endocardial and epicardial contours can be traced in one image frame and copied to other frames. Subsequently, time-intensity curves for particular myocardial regions can be easily generated. Although these curves can be determined at a pixel level, the noise level in the images is often not sufficient to derive reliable perfusion indices at this level of detail. More typically 4-8 segments are defined for each imaging section that can be further sub-divided into an endocardial and an epicardial layer [51].

\section{Absolute perfusion quantification}

In contrast to the semi-quantitative methods, models exist that quantify MBF. It may be important to 

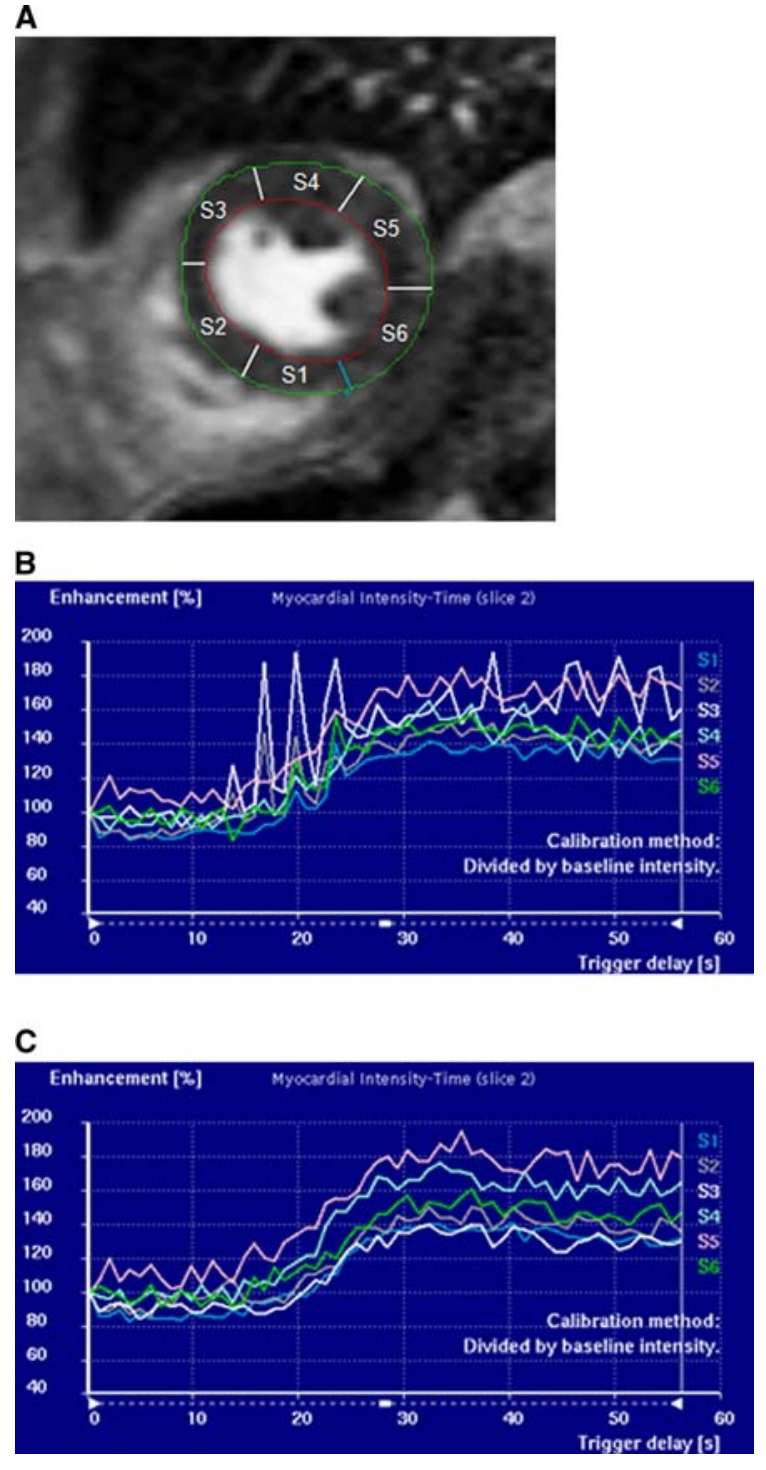

Fig. 2 Short-axis image at a mid ventricular slice level with endo- and epi-cardial contours defined and the myocardium divided into 6 segments (a). Signal-intensity versus time curves are derived for each of the myocardial segments. Without motion correction (b) the curves are not suitable for quantitative analysis. After motion correction (c), perfusion indices such as maximum upslope can be derived reliably

quantify myocardial perfusion and perfusion reserve in patients with multi-vessel disease because perfusion is often globally reduced and qualitative or semiquantitative measures are inadequate. Quantitative myocardial perfusion CMR studies have provided insights into the pathophysiology of epicardial coronary artery disease, microvascular disease and cardiomyopathy. The benefits of absolute blood flow quantification with CMR may improve clinical diagnosis and have important implications for cardiovascular research.

Several studies have evaluated perfusion in instrumented animals to compare noninvasive MBF by CMR with flow measured by injected microspheres, which provide a pathological gold standard for regional tissue perfusion [46, 52-54]. The overall correlation between CMR and microsphere MBF is good to excellent, with the correlation coefficient ranging from 0.79 to 0.95 over a wide range of myocardial blood flow. In addition, absolute perfusion correlated more closely with microsphere blood flow than established semi-quantitative CMR indexes [46]. In healthy volunteers MBF measurements by CMR have been found to be in agreement with published values based on invasive and noninvasive methods, and the magnitude of flow heterogeneity is similar to that seen in PET [55-57]. A significant correlation has been shown for both dipyridamoleinduced flow $(r=0.70, \quad P=0.001)$ and MPR $(r=0.48, P=0.04)$ between CMR and PET in healthy volunteers [58].

Absolute quantitative CMR perfusion has been recently used in a number of clinical studies in both patients with CAD and cardiomyopathy [59-65]. The myocardial perfusion reserve index, using quantitative CMR perfusion, has been shown to be reduced in the presence of early myonecrosis after percutaneous coronary intervention (PCI), using delayed enhancement MR (DE-MR) as the reference standard [64]. Hibernating myocardial segments demonstrate reduced resting myocardial blood flow on quantitative CMR perfusion imaging [65]. Percutaneous treatment of chronic total coronary occlusions improves regional hyperemic myocardial blood flow as measured by quantitative CMR perfusion imaging [59]. The fractional flow reserve (FFR), determined using invasive intracoronary pressure wires, is considered to be a reliable stenosis-specific method for determining hemodynamically significant CAD [66]. In a recent study an absolute quantitative CMR, a perfusion reserve cutoff of 2.04 was demonstrated to be $92.9 \%$ sensitive and $56.7 \%$ specific in predicting a coronary segment with FFR $\leq 0.75$ [60]. Quantitative perfusion CMR is thus potentially a safe noninvasive test that represents a stenosis-specific alternative for determining the hemodynamic significance of CAD. In hypertrophic cardiomyopathy 
(HCM), hyperemic blood flow, as measured by quantitative CMR perfusion, has been shown to be reduced in proportion to the magnitude of the hypertrophy [62]. Microvascular dysfunction and subsequent ischemia may be important components of the risk attributable to HCM.

Further studies are underway in the field of CMR perfusion imaging acquisition. Most contemporary pulse sequences do not achieve full heart coverage but can acquire at least three short-axis slices every heart beat with an acquisition time of approximately $150 \mathrm{~ms} / \mathrm{slice}$ and an in-plane spatial resolution of 2-3 mm. Faster pulse sequences, new acceleration techniques $[67,68]$ imaging at higher field strengths [69] and the use of intravascular contrast agents are under investigation with the potential to enable greater heart coverage, spatial resolution and or increased signal-to-contrast ratio.

\section{Delayed contrast enhanced magnetic resonance imaging}

DE-MR enables detection, characterization and accurate quantification of acute and chronic myocardial infarction. Quantification of infarct size with DE-MR has been validated against true infarct size as verified by histochemical staining with triphenyl tertazolium chloride (TTC) in animal models [70]. Acute infarct size, on DE-MR, directly relates to LV remodeling and is a stronger predictor of future events than measures of LV systolic performance [71, 72]. The transmural extent of the infarction as seen on DE-MR in chronic MI is inversely related to the likelihood of recovery following revascularization [73]. Infarct size on DE-MR has been shown to be superior to LVEF and LV volume for predicting long-term mortality in patients with healed myocardial infarction [74]. Myocardial scar forms a substrate for ventricular tachy-arrhythmias and scar quantification by DE-MR has the potential for allowing better identification of at-risk patients. In a study of patients with established CAD that underwent electrophysiological study (EPS) based sudden cardiac death (SCD) risk stratification, Bello et al. found that the infarct surface area $\left(172 \pm 15\right.$ vs. $\left.93 \pm 14 \mathrm{~cm}^{2}\right)$ and mass ( $49 \pm 5$ vs. $28 \pm 5 \mathrm{~g}$ ), as measured by DE-MR, were greater among patients that had sustained ventricular tachycardia (VT) compared to patients that were non inducible [75]. Another potentially important pathophysiological substrate for adverse post MI events is the peri-infarct zone as measured by DE-MR. Yan and colleagues found that the presence of extensive peri-infarct regions of intermediate hyperenhancement (defined by hyperenhancement with signal intensity two to three SD above normal) conferred an increased mortality risk [76]. The total scar burden, assessed using DE-MR, has been found to predict the clinical response to cardiac resynchronization therapy (CRT) [77, 78], and it may aid in better patient selection. In addition to allowing detection and characterization of the non-ischemic cardiomyopathies, scar quantification with DE-MR may play a role in determining prognosis and risk assessment. HCM patients with ventricular tachycardia have been found to have a higher percentage of myocardial scarring noted on DE-MR independent of septal thickness or beta blocker usage [79].

A 2D segmented inversion recovery GRE sequence is the standard sequence used for DE-MR [80]. Recently, an ultrafast, real-time version of DE-MR has become available and can acquire snapshot images during free breathing, although with lower spatial resolution and less T1 weighting [81]. The technique uses an inversion recovery steady-state free precession (SSFP) imaging sequence in singleshot mode with parallel imaging and provides complete left ventricular coverage in less than $30 \mathrm{~s}$. High accuracy for the detection of a MI has been shown and the technique can be considered the preferred approach in patients who are more acutely ill, unable to hold breath, or have irregular heart rhythms. Quantitative analysis however demonstrates moderately reduced contrast-to-noise ratios for subsecond imaging between the infarct and remote myocardium and between infarct and left ventricular cavity. Compared to standard imaging, the sensitivity is mildly reduced, and the transmural extent of the infarction may be underestimated by the single shot SSFP technique.

\section{Image processing for DE-MR imaging studies}

Various approaches have been suggested for determining the optimal intensity threshold characterizing the region of scar within the myocardium. In the pioneering work of Kim et al. hyperenhanced regions 
were defined as those regions having an intensity value $>2 \mathrm{D}$ above the mean of the remote normal myocardium [70]. Other investigators have suggested slight modifications to this approach by proposing the addition of two to five times the standard deviation $[82,83]$. These observations demonstrate the sensitivity of the SD-method to differences in the image acquisition protocol. As an alternative, the Full-WidthHalf-Maximum has been proposed and has been validated in animal studies by Amado and coworkers [84]. The inherent properties of the Full-Width-HalfMaximum method make it much less sensitive to variations in image acquisition parameters. Based on this criterion Hsu et al. developed a fully automated technique to obtain accurate assessment of the size of a
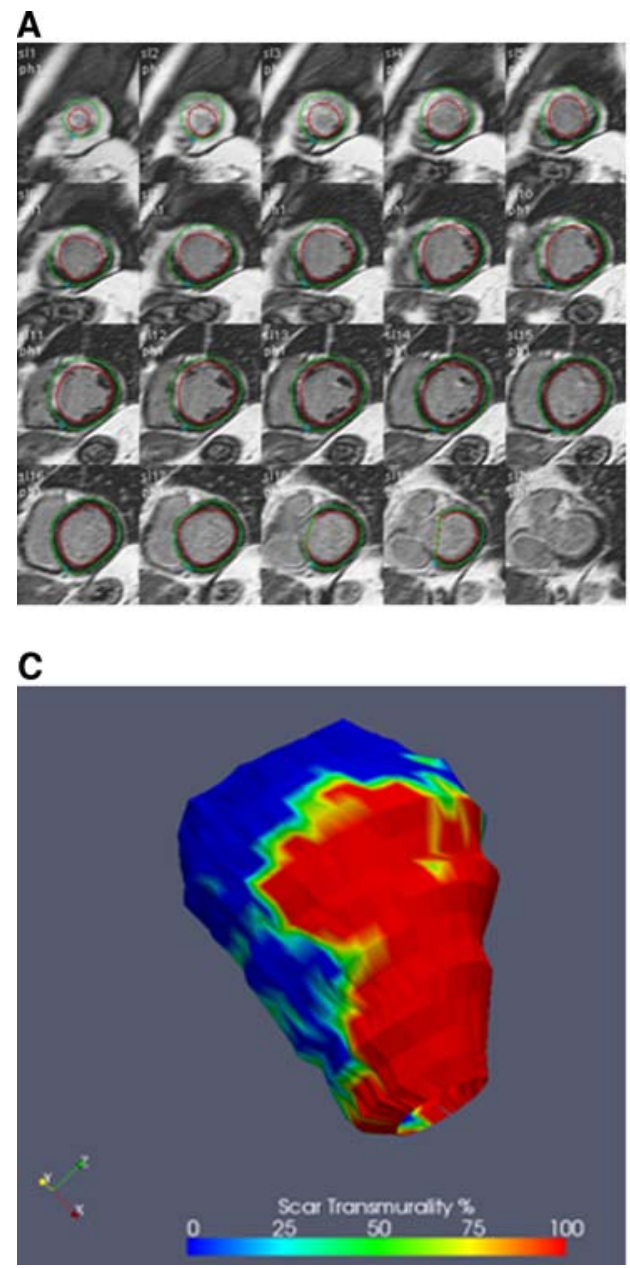

Fig. 3 a Multi-slice short-axis DEMR with defined endocardial and epicardial contours superimposed. Scar transmurality defined as the thickness of scar relative to the local wall myocardial infarction and validated this approach in an animal experimental setting [85]. The size of myocardial scar can easily be derived by counting the number of pixels within the myocardium above the defined threshold, which can be expressed in grams or as percentage of the LV myocardium. The local transmurality can be derived as the thickness of the scar relative to the local wall thickness (Fig. 3).

\section{Integrated analysis}

The increasing routine clinical use of CMR and the overwhelming size of the typical CMR image data sets pose a significant challenge for time-efficient

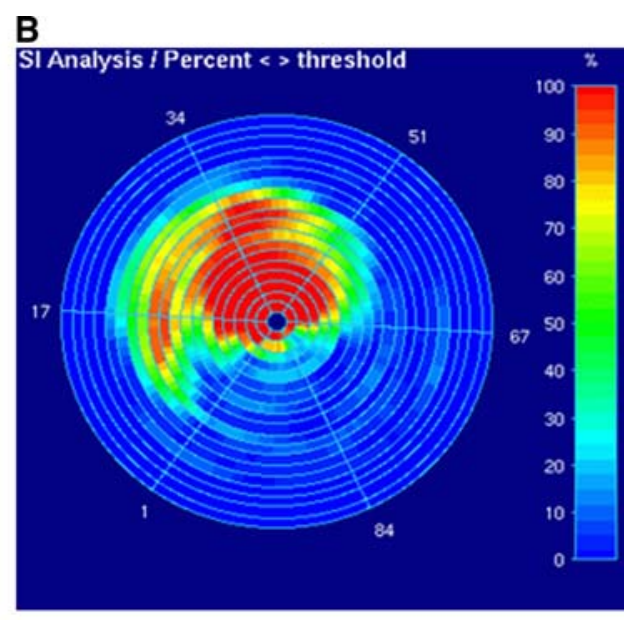

D

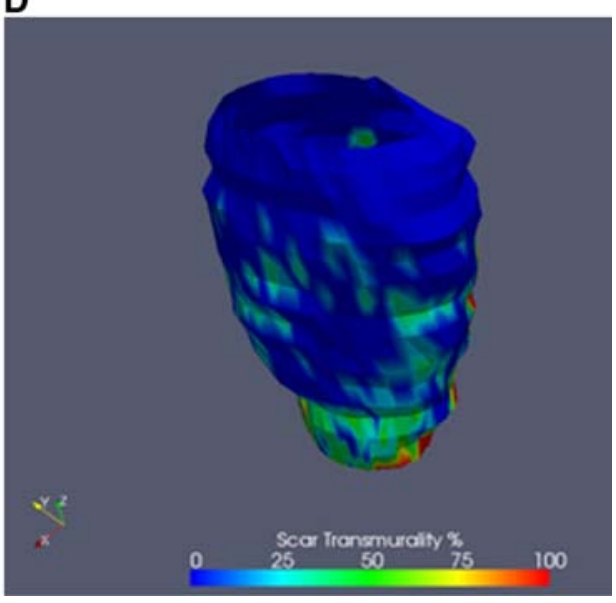

thickness assessed from DEMR. Scar distribution can be displayed as a bulls-eye plot (b), or as three-dimensional reconstructions $(\mathbf{c}, \mathbf{d})$ 
image quantification and interpretation. In the last decade various image analysis software packages with automated image segmentation and quantification methods have been introduced to support the work of the clinician. However, these software packages primarily focus on particular elements within a CMR exam, such as the assessment of global function or myocardial perfusion. The integration of the available post-processing techniques for CMR image analysis into a software solution for the analysis of all of the data acquired in a comprehensive CMR examination would be a major step forward. The work by Hennemuth et al. shows a significant step towards such an integrated image analysis solution [86].

Open Access This article is distributed under the terms of the Creative Commons Attribution Noncommercial License which permits any noncommercial use, distribution, and reproduction in any medium, provided the original author(s) and source are credited.

\section{References}

1. Pennell DJ, Sechtem UP, Higgins CB et al (2004) Clinical indications for cardiovascular magnetic resonance (CMR): Consensus Panel report. Eur Heart J 25:1940-1965

2. Pruessmann KP, Weiger M, Scheidegger MB et al (1999) SENSE: sensitivity encoding for fast MRI. Magn Reson Med 42:952-962

3. Griswold MA, Jakob PM, Heidemann RM et al (2002) Generalized autocalibrating partially parallel acquisitions (GRAPPA). Magn Reson Med 47:1202-1210

4. Kacere RD, Pereyra M, Nemeth MA et al (2005) Quantitative assessment of left ventricular function: steady-state free precession MR imaging with or without sensitivity encoding. Radiology 235:1031-1035

5. Tsao J, Boesiger P, Pruessmann KP (2003) k-t BLAST and k-t SENSE: dynamic MRI with high frame rate exploiting spatiotemporal correlations. Magn Reson Med 50:1031-1042

6. Greil GF, Germann S, Kozerke S et al (2008) Assessment of left ventricular volumes and mass with fast $3 \mathrm{D}$ cine steady-state free precession k-t space broad-use linear acquisition speed-up technique (k-t BLAST). J Magn Reson Imaging 27:510-515

7. Jahnke C, Nagel E, Gebker R et al (2007) Four-dimensional single breathhold magnetic resonance imaging using kt-BLAST enables reliable assessment of left- and rightventricular volumes and mass. J Magn Reson Imaging 25:737-742

8. Jahnke C, Paetsch I, Gebker R et al (2006) Accelerated 4D dobutamine stress MR imaging with k-t BLAST: feasibility and diagnostic performance. Radiology 241:718-728
9. Hardy CJ, Cline HE, Giaquinto RO et al (2006) 32-Element receiver-coil array for cardiac imaging. Magn Reson Med 55:1142-1149

10. Gerretsen SC, Versluis B, Bekkers SC et al (2008) Cardiac cine MRI: comparison of $1.5 \mathrm{~T}$, non-enhanced $3.0 \mathrm{~T}$ and blood pool enhanced 3.0 T imaging. Eur J Radiol 65:80-85

11. Sandner TA, Houck P, Runge VM et al (2008) Accuracy of accelerated cine MR imaging at 3 tesla in longitudinal follow-up of cardiac function. Eur Radiol 18:2095-2101

12. Sievers B, Brandts B, Franken U et al (2004) Single and biplane TrueFISP cardiovascular magnetic resonance for rapid evaluation of left ventricular volumes and ejection fraction. J Cardiovasc Magn Reson 6:593-600

13. Rogers WJ Jr, Shapiro EP, Weiss JL et al (1991) Quantification of and correction for left ventricular systolic longaxis shortening by magnetic resonance tissue tagging and slice isolation. Circulation 84:721-731

14. Alfakih K, Plein S, Bloomer T et al (2003) Comparison of right ventricular volume measurements between axial and short axis orientation using steady-state free precession magnetic resonance imaging. J Magn Reson Imaging 18: 25-32

15. van Assen HC, Danilouchkine MG, Frangi AF et al (2006) SPASM: a 3D-ASM for segmentation of sparse and arbitrarily oriented cardiac MRI data. Med Image Anal 10: 286-303

16. Mitchell SC, Lelieveldt BP, van der Geest RJ et al (2001) Multistage hybrid active appearance model matching: segmentation of left and right ventricles in cardiac MR images. IEEE Trans Med Imaging 20:415-423

17. Stegmann MB, Ersboll BK, Larsen R (2003) FAME-a flexible appearance modeling environment. IEEE Trans Med Imaging 22:1319-1331

18. van der Geest RJ, Lelieveldt BP, Angelie E et al (2004) Evaluation of a new method for automated detection of left ventricular boundaries in time series of magnetic resonance images using an Active Appearance Motion Model. J Cardiovasc Magn Reson 6:609-617

19. Cocosco CA, Niessen WJ, Netsch T et al (2008) Automatic image-driven segmentation of the ventricles in cardiac cine MRI. J Magn Reson Imaging 28:366-374

20. Codella NC, Weinsaft JW, Cham MD et al (2008) Left ventricle: automated segmentation by using myocardial effusion threshold reduction and intravoxel computation at MR imaging. Radiology 248:1004-1012

21. Zerhouni EA, Parish DM, Rogers WJ et al (1988) Human heart: tagging with MR imaging — a method for noninvasive assessment of myocardial motion. Radiology 169:59-63

22. Axel L, Dougherty L (1989) MR imaging of motion with spatial modulation of magnetization. Radiology 171:841-845

23. Fischer SE, McKinnon GC, Maier SE et al (1993) Improved myocardial tagging contrast. Magn Reson Med 30:191-200

24. Stuber M, Spiegel MA, Fischer SE et al (1999) Single breath-hold slice-following CSPAMM myocardial tagging. MAGMA 9:85-91

25. Ryf S, Schwitter J, Spiegel MA et al (2005) Accelerated tagging for the assessment of left ventricular myocardial contraction under physical stress. J Cardiovasc Magn Reson 7:693-703 
26. Herzka DA, Guttman MA, McVeigh ER (2003) Myocardial tagging with SSFP. Magn Reson Med 49:329-340

27. Kuijer JP, Hofman MB, Zwanenburg JJ et al (2006) DENSE and HARP: two views on the same technique of phase-based strain imaging. J Magn Reson Imaging 24:1432-1438

28. Osman NF, Sampath S, Atalar E et al (2001) Imaging longitudinal cardiac strain on short-axis images using strain-encoded MRI. Magn Reson Med 46:324-334

29. Neizel M, Lossnitzer D, Korosoglou G et al (2009) Strainencoded (SENC) magnetic resonance imaging to evaluate regional heterogeneity of myocardial strain in healthy volunteers: comparison with conventional tagging. J Magn Reson Imaging 29:99-105

30. Garot J, Lima JA, Gerber BL et al (2004) Spatially resolved imaging of myocardial function with strainencoded MR: comparison with delayed contrast-enhanced MR imaging after myocardial infarction. Radiology 233:596-602

31. Korosoglou G, Futterer S, Humpert PM et al (2009) Strainencoded cardiac MR during high-dose dobutamine stress testing: comparison to cine imaging and to myocardial tagging. J Magn Reson Imaging 29:1053-1061

32. Pan L, Stuber M, Kraitchman DL et al (2006) Real-time imaging of regional myocardial function using fast-SENC. Magn Reson Med 55:386-395

33. Korosoglou G, Youssef AA, Bilchick KC et al (2008) Real-time fast strain-encoded magnetic resonance imaging to evaluate regional myocardial function at 3.0 tesla: comparison to conventional tagging. J Magn Reson Imaging 27:1012-1018

34. Hamdan A, Thouet T, Kelle S et al (2008) Regional right ventricular function and timing of contraction in healthy volunteers evaluated by strain-encoded MRI. J Magn Reson Imaging 28:1379-1385

35. Youssef A, Ibrahim el SH, Korosoglou G et al (2008) Strain-encoding cardiovascular magnetic resonance for assessment of right-ventricular regional function. J Cardiovasc Magn Reson 10:33

36. Chalil S, Stegemann B, Muhyaldeen SA et al (2007) Effect of posterolateral left ventricular scar on mortality and morbidity following cardiac resynchronization therapy. Pacing Clin Electrophysiol 30:1201-1209

37. Marsan NA, Westenberg JJ, Ypenburg C et al (2009) Magnetic resonance imaging and response to cardiac resynchronization therapy: relative merits of left ventricular dyssynchrony and scar tissue. Eur Heart J 30:23602367

38. Markl M, Alley MT, Pelc NJ (2003) Balanced phasecontrast steady-state free precession (PC-SSFP): a novel technique for velocity encoding by gradient inversion. Magn Reson Med 49:945-952

39. Markl M, Chan FP, Alley MT et al (2003) Time-resolved three-dimensional phase-contrast MRI. J Magn Reson Imaging 17:499-506

40. Westenberg JJ, Roes SD, Ajmone Marsan N et al (2008) Mitral valve and tricuspid valve blood flow: accurate quantification with $3 \mathrm{D}$ velocity-encoded MR imaging with retrospective valve tracking. Radiology 249:792-800

41. Nandalur KR, Dwamena BA, Choudhri AF et al (2007) Diagnostic performance of stress cardiac magnetic resonance imaging in the detection of coronary artery disease: a meta-analysis. J Am Coll Cardiol 50:1343-1353

42. Schwitter J, Wacker CM, van Rossum AC et al (2008) MR-IMPACT: comparison of perfusion-cardiac magnetic resonance with single-photon emission computed tomography for the detection of coronary artery disease in a multicentre, multivendor, randomized trial. Eur Heart J 29:480-489

43. Jahnke C, Nagel E, Gebker R et al (2007) Prognostic value of cardiac magnetic resonance stress tests: adenosine stress perfusion and dobutamine stress wall motion imaging. Circulation 115:1769-1776

44. Gebker R, Schwitter J, Fleck E et al (2007) How we perform myocardial perfusion with cardiovascular magnetic resonance. J Cardiovasc Magn Reson 9:539-547

45. Gebker R, Paetsch I, Neuss M et al (2005) Determinants of myocardial response in CMR perfusion imaging using GdBOPTA (Multihance). J Cardiovasc Magn Reson 7:565-572

46. Christian TF, Aletras AH, Arai AE (2008) Estimation of absolute myocardial blood flow during first-pass MR perfusion imaging using a dual-bolus injection technique: comparison to single-bolus injection method. J Magn Reson Imaging 27:1271-1277

47. Nagel E, Klein C, Paetsch I et al (2003) Magnetic resonance perfusion measurements for the noninvasive detection of coronary artery disease. Circulation 108:432-437

48. Adluru G, Dibella EV (2008) Reordering for improved constrained reconstruction from undersampled k-space data. Int J Biomed Imaging 2008:341684

49. Bidaut LM, Vallee JP (2001) Automated registration of dynamic MR images for the quantification of myocardial perfusion. J Magn Reson Imaging 13:648-655

50. Milles J, van der Geest RJ, Jerosch-Herold M et al (2008) Fully automated motion correction in first-pass myocardial perfusion MR image sequences. IEEE Trans Med Imaging 27:1611-1621

51. Schwitter J, Nanz D, Kneifel S et al (2001) Assessment of myocardial perfusion in coronary artery disease by magnetic resonance: a comparison with positron emission tomography and coronary angiography. Circulation 103:2230-2235

52. Christian TF, Rettmann DW, Aletras AH et al (2004) Absolute myocardial perfusion in canines measured by using dual-bolus first-pass MR imaging. Radiology 232:677-684

53. Jerosch-Herold M, Swingen C, Seethamraju RT (2002) Myocardial blood flow quantification with MRI by modelindependent deconvolution. Med Phys 29:886-897

54. Muhling OM, Wang Y, Panse P et al (2003) Transmyocardial laser revascularization preserves regional myocardial perfusion: an MRI first pass perfusion study. Cardiovasc Res 57:63-70

55. Hsu LY, Rhoads KL, Holly JE et al (2006) Quantitative myocardial perfusion analysis with a dual-bolus contrastenhanced first-pass MRI technique in humans. J Magn Reson Imaging 23:315-322

56. Lee DC, Johnson NP (2009) Quantification of absolute myocardial blood flow by magnetic resonance perfusion imaging. JACC Cardiovasc Imaging 2:761-770

57. Muehling OM, Jerosch-Herold M, Panse P et al (2004) Regional heterogeneity of myocardial perfusion in healthy 
human myocardium: assessment with magnetic resonance perfusion imaging. J Cardiovasc Magn Reson 6:499-507

58. Parkka JP, Niemi P, Saraste A et al (2006) Comparison of MRI and positron emission tomography for measuring myocardial perfusion reserve in healthy humans. Magn Reson Med 55:772-779

59. Cheng AS, Selvanayagam JB, Jerosch-Herold $M$ et al (2008) Percutaneous treatment of chronic total coronary occlusions improves regional hyperemic myocardial blood flow and contractility: insights from quantitative cardiovascular magnetic resonance imaging. JACC Cardiovasc Interv 1:44-53

60. Costa MA, Shoemaker S, Futamatsu H et al (2007) Quantitative magnetic resonance perfusion imaging detects anatomic and physiologic coronary artery disease as measured by coronary angiography and fractional flow reserve. J Am Coll Cardiol 50:514-522

61. Muehling O, Jerosch-Herold M, Cyran C et al (2007) Assessment of collateralized myocardium with Cardiac Magnetic Resonance (CMR): transmural extent of infarction but not angiographic collateral vessel filling determines regional function and perfusion in collateral-dependent myocardium. Int J Cardiol 120:38-44

62. Petersen SE, Jerosch-Herold M, Hudsmith LE et al (2007) Evidence for microvascular dysfunction in hypertrophic cardiomyopathy: new insights from multiparametric magnetic resonance imaging. Circulation 115:2418-2425

63. Rivard AL, Swingen CM, Blake D et al (2007) A comparison of myocardial perfusion and rejection in cardiac transplant patients. Int J Cardiovasc Imaging 23:575-582

64. Selvanayagam JB, Cheng AS, Jerosch-Herold M et al (2007) Effect of distal embolization on myocardial perfusion reserve after percutaneous coronary intervention: a quantitative magnetic resonance perfusion study. Circulation 116:1458-1464

65. Selvanayagam JB, Jerosch-Herold M, Porto I et al (2005) Resting myocardial blood flow is impaired in hibernating myocardium: a magnetic resonance study of quantitative perfusion assessment. Circulation 112:3289-3296

66. Pijls NH, van Schaardenburgh P, Manoharan G et al (2007) Percutaneous coronary intervention of functionally nonsignificant stenosis: 5-year follow-up of the DEFER Study. J Am Coll Cardiol 49:2105-2111

67. Plein S, Kozerke S, Suerder D et al (2008) High spatial resolution myocardial perfusion cardiac magnetic resonance for the detection of coronary artery disease. Eur Heart J 29:2148-2155

68. Plein S, Ryf S, Schwitter J et al (2007) Dynamic contrastenhanced myocardial perfusion MRI accelerated with k-t sense. Magn Reson Med 58:777-785

69. Plein S, Schwitter J, Suerder D et al (2008) k-Space and time sensitivity encoding-accelerated myocardial perfusion $\mathrm{MR}$ imaging at $3.0 \mathrm{~T}$ : comparison with $1.5 \mathrm{~T}$. Radiology 249:493-500

70. Kim RJ, Fieno DS, Parrish TB et al (1999) Relationship of MRI delayed contrast enhancement to irreversible injury, infarct age, and contractile function. Circulation 100:19922002

71. Lund GK, Stork A, Muellerleile K et al (2007) Prediction of left ventricular remodeling and analysis of infarct resorption in patients with reperfused myocardial infarcts by using contrast-enhanced MR imaging. Radiology 245:95-102

72. Wu E, Ortiz JT, Tejedor P et al (2008) Infarct size by contrast enhanced cardiac magnetic resonance is a stronger predictor of outcomes than left ventricular ejection fraction or end-systolic volume index: prospective cohort study. Heart 94:730-736

73. Kim RJ, Wu E, Rafael A et al (2000) The use of contrastenhanced magnetic resonance imaging to identify reversible myocardial dysfunction. N Engl J Med 343: 1445-1453

74. Roes SD, Kelle S, Kaandorp TA et al (2007) Comparison of myocardial infarct size assessed with contrast-enhanced magnetic resonance imaging and left ventricular function and volumes to predict mortality in patients with healed myocardial infarction. Am J Cardiol 100:930-936

75. Bello D, Fieno DS, Kim RJ et al (2005) Infarct morphology identifies patients with substrate for sustained ventricular tachycardia. J Am Coll Cardiol 45:1104-1108

76. Yan AT, Shayne AJ, Brown KA et al (2006) Characterization of the peri-infarct zone by contrast-enhanced cardiac magnetic resonance imaging is a powerful predictor of post-myocardial infarction mortality. Circulation 114:32-39

77. White JA, Yee R, Yuan X et al (2006) Delayed enhancement magnetic resonance imaging predicts response to cardiac resynchronization therapy in patients with intraventricular dyssynchrony. J Am Coll Cardiol 48:19531960

78. Ypenburg C, Roes SD, Bleeker GB et al (2007) Effect of total scar burden on contrast-enhanced magnetic resonance imaging on response to cardiac resynchronization therapy. Am J Cardiol 99:657-660

79. Kwon DH, Setser RM, Popovic ZB et al (2008) Association of myocardial fibrosis, electrocardiography and ventricular tachyarrhythmia in hypertrophic cardiomyopathy: a delayed contrast enhanced MRI study. Int J Cardiovasc Imaging 24:617-625

80. Kim RJ, Shah DJ, Judd RM (2003) How we perform delayed enhancement imaging. J Cardiovasc Magn Reson 5:505-514

81. Sievers B, Elliott MD, Hurwitz LM et al (2007) Rapid detection of myocardial infarction by subsecond, freebreathing delayed contrast-enhancement cardiovascular magnetic resonance. Circulation 115:236-244

82. Beek AM, Bondarenko O, Afsharzada F et al (2009) Quantification of late gadolinium enhanced CMR in viability assessment in chronic ischemic heart disease: a comparison to functional outcome. J Cardiovasc Magn Reson 11:6

83. Bondarenko O, Beek AM, Hofman MB et al (2005) Standardizing the definition of hyperenhancement in the quantitative assessment of infarct size and myocardial viability using delayed contrast-enhanced CMR. J Cardiovasc Magn Reson 7:481-485

84. Amado LC, Gerber BL, Gupta SN et al (2004) Accurate and objective infarct sizing by contrast-enhanced magnetic resonance imaging in a canine myocardial infarction model. J Am Coll Cardiol 44:2383-2389 
85. Hsu LY, Natanzon A, Kellman P et al (2006) Quantitative myocardial infarction on delayed enhancement MRI. Part I: animal validation of an automated feature analysis and combined thresholding infarct sizing algorithm. J Magn Reson Imaging 23:298-308
86. Hennemuth A, Seeger A, Friman O et al (2008) A comprehensive approach to the analysis of contrast enhanced cardiac MR images. IEEE Trans Med Imaging 27:15921610 